\title{
Diseño y validación de una escala de relevancia competencial motivacional del libro de texto de ciencias ${ }^{1}$
}

\section{Design and Validation of the Motivational Competency Relevance Scale of Science Textbooks ${ }^{2}$ \\ Design e validação da escala de relevância de competência motivacional do livro de texto de ciências ${ }^{3}$}

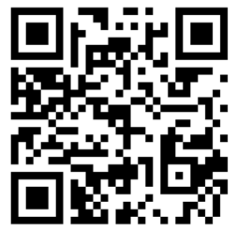

Raquel Romero-Fernández Universidad de Huelva Huelva, España raquel.romero@pi.uhu.es

https://orcid.org/0000-0002-9559-9516

María de los Ángeles De las Heras-Pérez Universidad de Huelva Huelva, España

angeles.delasheras@ddcc.uhu.es Dttps://orcid.org/0000-0002-3640-8337

Pedro Sáenz-López-Buñuel Universidad de Huelva Huelva, España psaenz@uhu.es

https://orcid.org/0000-0002-2979-5842

Eduardo José Fernández-Ozcorta Centro de Estudios Universitarios Cardenal Spínola Sevilla, España ejfernandez@ceuandalucia.es https://orcid.org/0000-0001-9823-1306

Recibido • Received • Recebido: 26 / 01 / 2020

Corregido • Revised • Revisado: 23 / 03 / 2021

Aceptado • Accepted • Aprovado: 24 / 04 / 2021

\footnotetext{
${ }^{1}$ Este artículo responde a una investigación mayor titulada La motivación del alumnado de primaria y secundaria y los libros de texto de ciencias (Tesis doctoral).

${ }^{2}$ This article responds to a major investigation entitled The Motivation of Primary and Secondary Students and Science Textbooks (doctoral thesis).

${ }^{3}$ Este artigo responde a uma investigação ampliada intitulada: A motivação de estudantes do ensino fundamental e médio e os livros de ciências (Tese de doutorado).
} 
http://doi.org/10.15359/ree.25-2.17

http://www.una.ac.cr/educare

educare@una.ac.cr

\begin{abstract}
Resumen:
Introducción. La falta de motivación en la educación científica es cada vez más preocupante. Muchas y variadas investigaciones han intentado, desde el enfoque de la teoría de la autodeterminación, aproximarse a comprender mejor la motivación, mediante el estudio de diferentes variables contextuales. Entre estas variables, el libro de texto es un elemento especialmente relevante, dada su importante presencia en las aulas y su, en principio, carácter competencial que, supuestamente, lo convierte en un recurso motivador. Objetivo. Por ello, se ha pretendido diseñar y validar una escala para medir la relevancia que tiene el libro de texto de ciencias, desde esta perspectiva competencial motivacional, para el alumnado con edades comprendidas entre los 10 y 14 años. Metodología. El diseño se realizó tomando como referencia el marco teórico existente y para su validación se ha recurrido a una metodología de carácter cuantitativa, mediante una técnica de encuesta. Resultados. El análisis factorial exploratorio y confirmatorio reveló la existencia de un único factor y consiguió unos índices de ajuste adecuados. Asimismo, el instrumento mostró una adecuada consistencia interna, estabilidad temporal, fiabilidad compuesta y validez convergente. Finalmente, las correlaciones positivas y significativas obtenidas con las motivaciones educativas, así como a la inversa con la amotivación, evidenciaron la validez del instrumento externamente. Conclusiones. En consecuencia, se concluyó que la escala era válida y fiable.
\end{abstract}

Palabras claves: Validación de escala; motivación; competencia; libro de texto.

\begin{abstract}
:
Introduction. Lack of motivation in science education is increasingly worrying. From the Theory of Self-Determination, many and varied investigations have tried to approach a better understanding of motivation by studying different contextual variables. Among these variables, textbooks are especially relevant elements, given their important presence in classrooms and their, in principle, competence character that supposedly makes them a motivating resource. Objective. Therefore, the aim has been to design and validate a scale to measure the relevance of science textbooks, from this motivational competence perspective, for students aged between 10 and 14 years. Method. The design took as reference the existing theoretical framework and, for the validation, a quantitative methodology was implemented, using a survey technique. Results. The exploratory and confirmatory factor analysis revealed the existence of a single factor, achieving adequate adjustment rates. Likewise, the instrument showed adequate internal consistency, temporal stability, composite reliability, and convergent validity. Finally, the positive and significant correlations obtained with the educational motivations and inversely with demotivation evidenced the external validity of the instrument. Conclusions. Consequently, it was concluded that the scale was valid and reliable.
\end{abstract}

Keywords: Scale validation, motivation, skill, textbook.

\title{
Resumo:
}

Introdução. A situação de desmotivação na educação científica é cada vez mais preocupante. Muitas e variadas investigações tentaram, sob a abordagem da Teoria da Autodeterminação, aproximar um melhor entendimento da motivação, estudando diferentes variáveis contextuais. Dentre essas variáveis, o livro didático é um elemento especialmente relevante, dada sua presença importante nas salas de aula e seu caráter de competência que, supostamente, os torna um recurso motivador. Objetivo. Portanto, pretende-se projetar e validar uma escala para medir a relevância do livro de ciências, sob essa perspectiva de competência motivacional, para estudantes com idade entre 10 
e 14 anos. Metodologia. O delineamento foi realizado utilizando como referencial o marco teórico existente e, para sua validação, foi utilizada uma metodologia quantitativa, com uma técnica de pesquisa. Resultados. A análise fatorial exploratória e confirmatória revelou a existência de um único fator, atingindo taxas de ajuste adequadas. Além disso, o instrumento mostrou consistência interna adequada, estabilidade temporal, confiabilidade composta e validade convergente. Por fim, as correlações positivas e significativas obtidas com as motivações educacionais, bem como as contrarias como a motivação, evidenciaram a validade do instrumento externamente. Conclusão. Consequentemente, concluiu-se que a escala era válida e confiável.

Palavras-chave: Validação de escala; motivação; competência; livro de texto.

\section{Introducción}

El desinterés por el estudio de las ciencias está cada vez más extendido entre el estudiantado de los países desarrollados (Couso Lagarón et al., 2011). A su vez, el último informe español sobre los resultados PISA ha puesto de manifiesto que la puntuación media estimada en ciencias es la más baja que se ha obtenido del estudiantado en los últimos diez años, así como significativamente inferior a la media de la OCDE y al total de la UE (Ministerio de Educación y Formación Profesional, 2019). En consecuencia, la motivación se sitúa como elemento clave, ya que gran parte de las dificultades académicas se vinculan a la vertiente motivacional, la cual influye sobre la forma de pensar y, de esta manera, en el aprendizaje (Alonso Tapia y de la Red Fadrique, 2007). En concreto, la motivación puede ser definida como la dinamización del comportamiento en pos de un objetivo, por tanto es un elemento fundamental en la interacción con el mundo y entre las personas (Simpson y Balsan, 2016). Es tal la vinculación entre la motivación y la relación con el contexto, que el tipo de motivación presentada por una persona condiciona sus resultados académicos (Howard et al., 2021). En esta línea, el estudio realizado por Castro-Pérez y Morales-Ramírez (2015) puso de manifiesto que el tipo de actividades y los materiales didácticos son dos aspectos de gran relevancia para el alumnado con edades comprendidas entre los 9 y 12 años.

La teoría de la autodeterminación (Deci y Ryan, 1985; Ryan y Deci, 2019) se presenta como un amplio marco que ayuda a la comprensión de los factores que facilitan o socavan la motivación intrínseca, la motivación extrínseca autónoma y el bienestar psicológico, de todos los temas de relevancia directa en los entornos educativos (Ryan y Deci, 2020). En este sentido, considera que dicha motivación se encuentra influida por el clima motivacional, el cual comprende diferentes elementos, entre los que se encuentran las tareas escolares. A su vez, dichas tareas se ven influenciadas por las características de los materiales que utiliza el personal docente, entre los que se encuentran los libros de texto. Asimismo, la llegada de las competencias claves a la legislación educativa española debería haber provocado un enriquecimiento didáctico de los materiales escolares, ya que su diseño debe seguir criterios didácticos y pedagógicos, debido a su finalidad escolar, en la normativa vigente (Occelli y Valeiras, 2013). En concreto, los 
http://doi.org/10.15359/ree.25-2.17

http://www.una.ac.cr/educare

educare@una.ac.cr

manuales escolares deberían haberse impregnado de un enfoque en el que las competencias sean entendidas como "un poder actuar eficazmente en una clase de situaciones, movilizando y combinado en tiempo real y de forma pertinente recursos intelectuales y emocionales" (Perrenoud, 2012, p. 57), y en el que la motivación del alumnado sea un objetivo a conseguir, ya que es un aspecto clave e indispensable para desarrollar la competencia científica (Organización para la Cooperación y el Desarrollo Económicos [OCDE], 2006), debido a que se encuentra vinculada a la inteligencia emocional necesaria para tal desarrollo (Marco Stiefel, 2008).

En consecuencia, es necesario profundizar en el estudio de todos los aspectos potencialmente influyentes en la motivación, ya que esta es necesaria para el desarrollo competencial del alumnado. Por lo que, en este caso, se coloca el foco de atención sobre el libro de texto, provocando la necesidad del diseño de un instrumento válido y fiable que permita conocer su carácter motivador desde un enfoque competencial, ya que dicho carácter es relevante para el desarrollo de las competencias por parte del estudiantado. De esta forma, la herramienta facilitará en futuras investigaciones comprender el papel del libro de texto de ciencias en la motivación del estudiantado con un enfoque competencial. Así, finalmente ayudará a los docentes y editoriales a tomar las decisiones, y proponer las sugerencias didácticas adecuadas y fundamentadas para contribuir a la mejora del proceso de enseñanza-aprendizaje competencial desde una perspectiva motivacional.

\section{Marco teórico}

\section{Los manuales escolares como elemento contextual motivacional en el enfoque de la teoría de la autodeterminación}

De las miniteorías que componen la teoría de la autodeterminación, la miniteoría de integración orgánica se centra en las diferentes formas de motivación, así como en los factores contextuales que promueven o impiden la interiorización e integración de la regulación de la conducta motivada (Deci y Ryan, 1985). Los tipos de motivación son presentados como un continuo desde la forma menos a la más autodeterminada (Ryan y Deci, 2019). La amotivación, que se relaciona con la forma menos autodeterminada de conducta, es definida como el estado en el que no hay intención de actuar. En el extremo opuesto, la motivación intrínseca hace referencia al desarrollo de una actividad por la satisfacción inherente al realizarla. En concreto, Vallerand (1997) distingue entre la motivación intrínseca hacia el conocimiento, que se refiere a la realización de una actividad por el placer que se experimenta cuando se aprende, y la motivación intrínseca hacia el logro, donde la satisfacción se encuentra en el intentar superar un nuevo nivel. La regulación externa y la introyectada, ambas motivaciones extrínsecas y situadas en la zona intermedia, se relacionan con las conductas que se comienzan y controlan mediante una demanda externa o recompensa, en el primer caso, y con la realización de una acción para evitar la culpa o realzar su propio ego, en el segundo (Ryan y Deci, 2019). 
Asimismo, la interiorización es entendida como un proceso natural mediante el cual la persona transforma las peticiones o costumbres en valores personales y autorregulados, es decir, es la forma en que las regulaciones externas son asimiladas y reconstruidas, de manera que la conducta puede llegar a ser autodeterminada (Ryan y Deci, 2017). Si este proceso funciona de forma óptima, la persona se identificará con la importancia de las regulaciones sociales, asimilándolas e integrándolas como suyas y, por tanto, llegando a estar más integrada psicológica y socialmente. Sin embargo, el proceso puede no darse de forma completa, y provocar diferentes grados de interiorización de la regulación que se refleja en diferentes motivaciones (Ryan y Deci, 2000). La efectividad de este proceso de interiorización se encuentra influida por el contexto social (Ryan y Deci, 2017).

Un aspecto relevante es que el continuo de motivación no es subyacente a un continuo de desarrollo, ya que cada persona puede adoptar una regulación del comportamiento dependiendo de sus experiencias previas y entornos (Ryan, 1995). Así, estos estudios exponen que, aunque no hay una secuencia, sí existen razones que favorecen estos cambios. En concreto, ponen de manifiesto que las tareas que no presentan características como el ser interesantes, novedosas o desafiantes no se iniciarán a través de la motivación intrínseca (Deci y Ryan, 1985), y necesitan una motivación externa para iniciarlas y mantenerlas (Ryan y Deci, 2019). A su vez, la motivación intrínseca aumenta cuando las tareas presentan estas particularidades (Vallerand, 1997), aparece la amotivación cuando la actividad no es valorada (Ryan, 1995). Sin embargo, no detallan dónde reside el interés de la actividad, más allá de puntualizar que, incluyen dentro de estas actividades las que por término medio las personas consideran interesantes.

En este sentido, en el enfoque de la teoría de la autodeterminación (Deci y Ryan, 1985; Ryan y Deci, 2019), los libros de texto, donde se recogen gran cantidad de las tareas del alumnado, pueden ser considerados un elemento relevante del clima motivacional que influye en la motivación del alumnado y, por tanto, también en el desarrollo competencial de este (Marco Stiefel, 2008; OCDE, 2006). Sin embargo, la ausencia de una herramienta que nos aporte información sobre este recurso, desde una perspectiva competencial y motivacional, impide que se pueda valorar su influencia en el contexto motivacional del alumnado. Por ello, en el siguiente apartado se recogen diferentes aportaciones que realizan los estudios implicados en la investigación sobre la motivación en el contexto educativo; en este sentido, se convierten en el marco de referencia para el diseño del instrumento de investigación necesario.

\section{El libro de texto como elemento motivador en un enfoque competencial}

Muchas y variadas son las aportaciones que se han realizado a través de la investigación sobre la motivación en torno a las tareas escolares, por lo que, sin pretender recogerlas todas, se trata de abarcar una variedad considerable que ayude a concretar rasgos generales que puedan tener una influencia sobre la motivación en una perspectiva competencial. 
http://doi.org/10.15359/ree.25-2.17

http://www.una.ac.cr/educare

educare@una.ac.cr

La motivación, en el enfoque competencial que debería impregnar los libros de textos actuales, es un aspecto que se relaciona, entre otros elementos, con la curiosidad, es decir, con experimentar un sentimiento satisfactorio al buscar y conocer cosas (Marco Stiefel, 2008). En este sentido, según la OCDE (Ministerio de Educación y Formación Profesional, 2019), se vincula con la percepción del estudiantado sobre la relevancia de las ciencias en su vida diaria y su utilidad para continuar aprendiéndolas. Mientras que, los resultados obtenidos por Rubio Hurtado et al. (2016) destacan que el alumnado indica como útiles las actividades para desarrollar competencias aquellas que conllevan la simulación o aplicación de contenidos, interacción con otros alumnos y alumnas y facilitan aprobar la asignatura. Asimismo, se ha detectado que las propuestas con enfoque CTS que permitan establecer relaciones entre los contenidos y la vida diaria favorecerá el aprendizaje y la motivación del estudiantado (de Toledo y Camero, 2015). Así, la motivación puede verse favorecida mediante la conexión de los conocimientos de ciencias con el contexto social del estudiantado y haciendo explícita su relevancia (Couso Lagarón et al., 2011), ya que la falta de motivación podría relacionarse con la descontextualización de los temas respecto a la vida cotidiana (Busquets et al., 2016). En consecuencia, desde esta perspectiva, las tareas deben ser atractivas, que favorezcan el establecer relaciones entre lo que se conoce y lo que se espera aprender, y útiles para la vida (Ballester Vila y Sánchez Santamaría, 2011).

Asimismo, anteriormente a la llegada de las competencias, los estudios implicados en la investigación sobre la motivación en el contexto educativo han realizado diversas aportaciones sobre cómo favorecer la motivación del alumnado, de las cuales, algunas deben ser tenidas en cuenta por su estrecha cercanía al enfoque competencial motivacional.

Diferentes autores indican que el esfuerzo e interés con el que el alumnado realiza sus tareas depende, entre otros aspectos, del valor que les otorgan, al cual hacen referencia desde tres componentes (Pintrich, 1999): el interés intrínseco, su utilidad e importancia. El interés intrínseco hace referencia al placer obtenido cuando se produce la implicación en la tarea; la utilidad se relaciona con la importancia de la tarea para conseguir metas; y la importancia es vinculada con la importancia de hacer bien la tarea.

Ames (1992), como forma de favorecer la motivación, sugiere centrarse en un diseño basado en retos razonables, novedosos y variados. A su vez, Pozuelos Estrada (2007) propone la simulación de situaciones basadas en la realidad cercana al alumnado para poder aplicar sus conocimientos; mientras que Solbes et al. (2007) plantean que el alumnado propone, como formas de aumentar su interés, las relaciones ciencia-tecnología-sociedad, en lo que se coincide con los resultados obtenidos por Coronas Cabrero (1995) en los que se pone de manifiesto que el trabajar en actividades que relacionen el temario con los cambio científicos actuales promueve un mayor interés en el alumnado. 
http://doi.org/10.15359/ree.25-2.17

Por su parte, Cubero Pérez (2001) señala que mucho estudiantado afirma desconocer la razón por la cual es necesario trabajar una asignatura, por lo que su meta se traduce en un intento por superar el examen. En consecuencia, continuando con este autor, es necesario dotar de sentido lo que se pretende que el alumnado aprenda para que este valore su aprendizaje, mediante las aplicaciones en la vida diaria, las cuales ayudan a contextualizar y valorar lo aprendido. A su vez, Ugartetxea (2002) manifiesta que es importante que el alumnado considere que lo aprendido les ofrecerá nuevas posibilidades.

Por otro lado, un contenido interesante y relacionado con las necesidades del alumnado es necesario para que sea asumido por este, mediante la conexión entre las propuestas académicas y sus intereses iniciales, e intentar que este campo de interés inicial sea cada vez mayor (Cubero Pérez, 2001).

\section{Método}

\section{Problema de investigación}

Indagar acerca de los elementos motivacionales intervinientes en el trabajo competencial, entre los que destaca el libro de texto debido a su relevancia en las clases de ciencias, puede contribuir a la mejora de estrategias metodológicas que favorezcan el desarrollo competencial desde un enfoque motivacional. Así, el objetivo de este estudio es diseñar y validar una escala que permita medir la relevancia que tiene el libro de texto de ciencias a nivel motivacional desde una perspectiva competencial, para el alumnado del tercer ciclo de primaria y primer ciclo de secundaria. De acuerdo con esto, el problema que abordaremos en este artículo es: ¿Cómo medir la relevancia competencial motivacional de los libros de texto de ciencias entre un alumnado con edades comprendida entre los 10 y 14 años?

\section{Diseño de la investigación}

Esta investigación se ha desarrollado en dos fases consecutivas. En la primera fase se diseñó la escala y se llevó a cabo una prueba piloto con 63 participantes como primer paso del proceso de validación. Finalmente, en la segunda fase, la escala fue sometida a diversos análisis estadísticos con una muestra de 1000 participantes, con el objetivo de profundizar en el proceso de validación.

\section{Procedimiento}

El diseño de la escala se realizó tomando como referencia el marco teórico anteriormente referenciado. Los cuatros aspectos motivacionales, desde un enfoque competencial, citados bibliográficamente con mayor influencia en torno al libro de texto y que, por tanto, se seleccionaron para su medición fueron: 
http://doi.org/10.15359/ree.25-2.17

http://www.una.ac.cr/educare

educare@una.ac.cr

- Un contenido con interés para el alumnado.

- Utilidad de lo aprendido en el entorno más próximo.

- Un aprendizaje que suscite la curiosidad y la necesidad de continuar aprendiendo.

- Satisfacción de la curiosidad.

Cada uno de estos aspectos fueron reformulados para la construcción de la escala. En el caso de la selección de los ítems definitivos, en primer lugar, se llevó a cabo una validación de contenido mediante la valoración de la claridad del lenguaje y la relevancia para el constructo con una escala Likert de cinco puntos, por parte de tres personas expertas en motivación educativa y didáctica. Siguiendo a Abad et al. (2011), se tuvo en cuenta que debían mantenerse aquellos ítems cuya claridad del lenguaje y relevancia fueran altos, quedándose finalmente compuesta por 4 ítems.

Tras tener el instrumento preparado, se contactó con un centro educativo de la provincia de Huelva para llevar a cabo una prueba piloto. Este centro fue informado sobre el objetivo de la investigación y el procedimiento que se seguiría. Tras el consentimiento informado, uno de los investigadores se desplazó al centro para llevar a cabo el proceso. La selección de la muestra fue incidental, se tuvo en cuenta que estuvieran representados ambos niveles educativos que son objeto de estudio de esta investigación. Todos los sujetos participantes fueron debidamente informados y se les garantizó el anonimato. Asimismo, se les pidió que hicieran las aportaciones que vieran necesarias respecto a la comprensión y dificultad. Transcurridos 16 días se volvió a repetir el mismo procedimiento.

En el caso del segundo estudio, la selección de la muestra se realizó en la provincia de Huelva, con un muestreo no aleatorio y teniendo en cuenta un tamaño muestral como mínimo 10 veces mayor que el número de ítems (Velicer y Fava, 1998). A continuación, se contactó con los diferentes centros educativos y se les informó sobre el estudio. A todos los suketos participantes se les explicó la investigación, el procedimiento y se les garantizó el anonimato.

\section{Participantes}

En la primera fase se han obtenido datos de la escala en una muestra de 63 estudiantes, 38 del tercer ciclo de primaria y 25 del primer ciclo de secundaria.

La muestra de participantes en la segunda fase del proceso de validación estuvo compuesta por 1000 escolares, con edades comprendidas entre los 10 y 14 años, 475 del tercer ciclo de primaria y 525 del primer ciclo de secundaria. Dichas personas participantes procedían de 13 centros educativos de la provincia de Huelva. 
http://doi.org/10.15359/ree.25-2.17

\section{Instrumentos}

Los instrumentos utilizados en esta investigación han sido dos escalas. La primera de ellas, denominada "Escala para medir la relevancia competencial motivacional del libro de texto de ciencias (RCMLC)", es el instrumento que ha sido diseñado y se pretende validar con este estudio. Por otro lado, la "Escala de motivación educativa (EME)" ha sido utilizada para realizar el proceso de validación externo de la escala diseñada.

Escala para medir la relevancia competencial motivacional del libro de texto de ciencias (RCMLC). La escala se encuentra formada por 4 ítems precedidos por la frase El libro de ciencia, ... los cuales conforman un único constructo que mide la relevancia que otorga el alumnado a sus libros de texto de ciencias en relación con el desarrollo de las competencias desde una perspectiva motivacional. Las respuestas se realizaban a través de una escala tipo Likert de 1 (Totalmente en desacuerdo) a 5 (Totalmente en desacuerdo).

Escala de Motivación Educativa (EME). Para medir la motivación educativa se seleccionaron los factores más relevantes, teniendo en cuenta nuestro estudio, de la versión para postsecundaria de la Escala de motivación educativa (Núñez et al., 2010). La versión utilizada estaba formada por 7 sub-escalas, compuestas cada una por 4 ítems que se refieren a las razones por las que el estudiantado acude al instituto. De las 7 subescalas se seleccionaron 5, las cuales fueron: amotivación, regulación externa, regulación introyectada, motivación intrínseca al conocimiento y motivación intrínseca al logro. Así, la escala inicialmente quedó formada por 25 ítems y precedida por la sentencia Estudio porque..., respondiéndose mediante una escala tipo Likert de 1 (Totalmente en desacuerdo) a 5 (Totalmente en desacuerdo). En este caso los valores de alfa de Cronbach fueron: .74 para amotivación, .73 para regulación externa, .69 para regulación introyectada, 84 para motivación intrínseca hacia el conocimiento y .76 para motivación intrínseca hacia el logro.

\section{Técnicas de análisis}

En la primera fase del proceso de validación, la escala diseñada fue sometida a un análisis en el que se estudiaron las propiedades psicométricas del cuestionario para comprobar su consistencia interna y estabilidad temporal. Asimismo, se realizó un análisis factorial exploratorio para explorar la unidimensionalidad del constructo. El paquete estadístico utilizado fue el SPSS 22.0.

Durante la segunda fase, primeramente, se llevó a cabo un análisis factorial exploratorio. A continuación, para confirmar el modelo planteado, se realizó un análisis factorial confirmatorio, se evaluó la fiabilidad de este mediante la varianza extraída y la fiabilidad compuesta, la validez convergente y su consistencia interna. Asimismo, se calcularon los estadísticos descriptivos y las correlaciones bivariadas entre la relevancia competencial del libro de texto de ciencias y la motivación educativa para obtener evidencias externas de la validez de la escala diseñada. Todos estos análisis se realizaron con los paquetes estadísticos SPSS 22.0 y AMOS 22.0. 
http://doi.org/10.15359/ree.25-2.17

http://www.una.ac.cr/educare

educare@una.ac.cr

\section{Resultados}

\section{Resultados de la primera fase del proceso de validación}

La estabilidad temporal fue analizada mediante el procedimiento test-retest. La correlación entre el test y re-test fue de .57, esto es, se obtuvo una estabilidad temporal adecuada.

La consistencia interna fue medida mediante el coeficiente de alfa de Cronbach, el cual obtuvo un valor de .66, por lo que alcanzaba un valor considerable que nos permitía concluir que la escala discriminaba adecuadamente al estudiantado en la percepción de relevancia competencial motivacional del libro de texto.

Para evaluar la estructura factorial de la escala, se llevó a cabo un análisis factorial exploratorio mediante la técnica multivariante de análisis de componentes principales y rotación ortogonal con método Varimax. La medida de la adecuación muestral de Kaiser-MeyerOlkin (KMO) obtuvo un valor de .790 y el test de esfericidad de Bartlett resultó estadísticamente significativo $\left(X^{2}=82.378 ; \mathrm{p}<.001\right)$, por lo que la adecuación de nuestros datos a un modelo de análisis factorial era buena y podíamos rechazar la hipótesis nula de variables iniciales incorrelacionadas; se respalda, de esta manera, la aplicación del análisis factorial.

Se extrajo un único factor formado por los cuatro ítems a través del criterio de Kaiser, se ajustó, así, a la estructura inicial propuesta para el instrumento (Tabla 1). La varianza total explicada mostraba que los cuatros componentes resumían el $65.036 \%$ de la variabilidad total.

Tabla 1: Análisis factorial exploratorio de la escala para medir la relevancia competencial motivacional percibida del libro de texto de ciencias del estudio 1

\begin{tabular}{lr}
\hline \multicolumn{1}{c}{ Ítems } & Componente \\
\hline Cuando resuelvo las actividades del libro de texto siento que quiero conocer más cosas sobre el tema & .815 \\
Lo que aprendo con el libro de texto lo puedo utilizar en mi vida diaria & .772 \\
Las actividades del libro de texto tratan temas interesantes & .814 \\
Las actividades del libro de texto me ayudan a resolver mis preguntas sobre la naturaleza & .823 \\
Varianza explicada (\%) & 65.036 \\
\hline
\end{tabular}

Nota: Elaboración propia.

10 Raquel Romero-Fernández, María de los Ángeles De las Heras-Pérez, Pedro Sáenz-López-Buñuel y Eduardo J. Fernández-Ozcorta 
http://doi.org/10.15359/ree.25-2.17

\section{Resultados de la segunda fase del proceso de validación}

La consistencia interna de la escala relevancia competencial motivacional del libro de texto, medida a través del coeficiente de alfa de Cronbach, obtuvo en este caso un valor de .827 , por lo que nuevamente presentó un valor considerable que permitía deducir que la escala discriminaba adecuadamente al estudiantado en dicha escala.

En la Tabla 2 pueden verse los datos obtenidos del análisis factorial exploratorio, las medias y desviaciones típicas para los ítems de la escala en el Estudio 2. Como puede observarse, mediante la prueba KMO (.808) y el test de esfericidad de Bartlett $\left(X^{2}=1416.058 ; p<.001\right)$, se concluye que la muestra es adecuada para llevar a cabo la factorización de los ítems. El análisis factorial exploratorio, a través de la técnica multivariante de análisis de componentes principales y rotación ortogonal con método Varimax extrae, mediante el criterio de Kaiser, un único factor que aglutina los cuatro ítems; se coincide, así, con el modelo teórico. Este factor explica un 65.97 $\%$ de la varianza total.

Tabla 2: Estadísticos descriptivos y análisis factorial exploratorio de la escala para medir la relevancia competencial motivacional percibida del libro de texto de ciencias del estudio 2

\begin{tabular}{lccc}
\hline \multicolumn{1}{c}{ Ítems } & Media & Desv. Típ. & Componente \\
\hline $\begin{array}{l}\text { Cuando resuelvo las actividades del libro de texto siento que quiero } \\
\text { conocer más cosas sobre el tema }\end{array}$ & 3.367 & 1.249 & .822 \\
$\begin{array}{l}\text { Lo que aprendo con el libro de texto lo puedo utilizar en mi vida diaria } \\
\text { Las actividades del libro de texto tratan temas interesantes }\end{array}$ & 3.589 & 1.229 & .776 \\
$\begin{array}{l}\text { Las actividades del libro de texto me ayudan a resolver mis preguntas } \\
\text { sobre la naturaleza }\end{array}$ & 3.586 & 1.203 & .832 \\
Varianza explicada (\%) & & 1.780 & .818 \\
\hline
\end{tabular}

Nota: Elaboración propia.

La confirmación de la existencia de un único factor se realizó mediante un análisis factorial confirmatorio. El diagrama del modelo se muestra en la Figura 1, donde pueden observarse un único constructo latente que agrupa 4 medidas observadas. 
http://doi.org/10.15359/ree.25-2.17

http://www.una.ac.cr/educare

educare@una.ac.cr

Figura 1: Análisis factorial confirmatorio de la escala relevancia competencial motivacional del libro de texto de ciencias

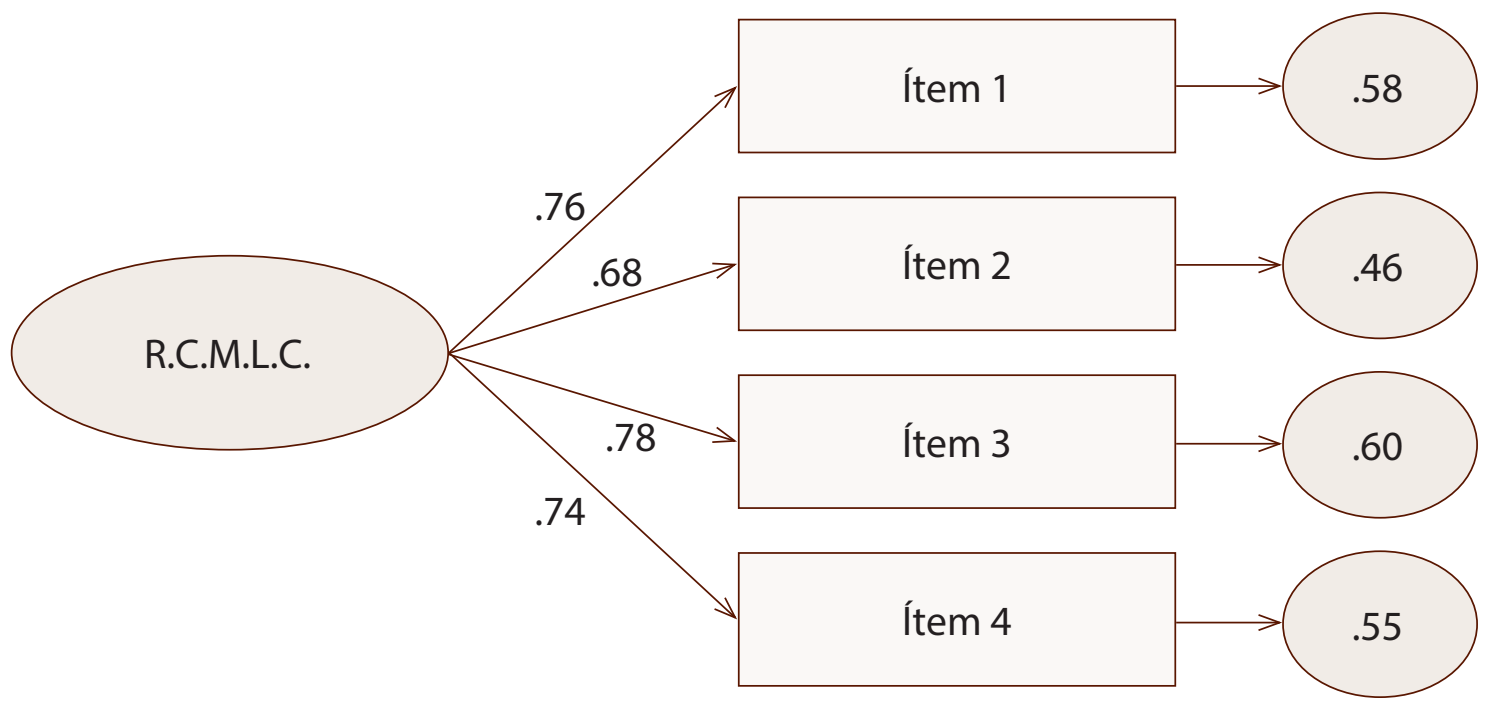

Nota: La elipse representa la variable latente y los rectángulos los diferentes ítems. Todos los parámetros se encuentran estandarizados y son estadísticamente significativos $(p<0.05)$. Los pequeños círculos recogen las varianzas error. RCMLC (Relevancia competencial motivacional del libro de texto de Ciencias). Elaboración propia.

Asimismo, con el objetivo de comprobar la validez del modelo, siguiendo las recomendaciones de Lévy Mangin y Varela Mallou (2006), se llevó a cabo una evaluación de la bondad de ajuste mediante la valoración complementaria de los índices más comunes atendiendo a los tres tipos de ajuste global (Tabla 3).

Tabla 3: Índices de bondad de ajuste del modelo ( $\mathrm{N}=1000)$

\begin{tabular}{cccccccc}
\hline \multirow{2}{*}{ MODELO } & \multicolumn{6}{c}{ ÍNDICES DE BONDAD DE AJUSTE } \\
\cline { 2 - 8 } & $X^{2}$ & X2/g.I & CFI & IFI & GFI & SRMR & RMSEA \\
\hline Un factor de primer orden con 4 indicadores & 5.312 & 2.66 & .99 & .99 & .99 & .011 & .041 \\
\hline
\end{tabular}

Nota: Elaboración propia.

En concreto, siguiendo con estos mismos estudios, los índices incrementales (CFI, IFI) son considerados aceptables cuando muestran valores superiores a .95, por lo que en este caso son satisfactorios al obtener un valor de .99 para ambos índices. Por su parte, los índices de error (RMSEA y SRMR) los consideran aceptables con valores de .05 o inferiores. En consecuencia,

12 Raquel Romero-Fernández, María de los Ángeles De las Heras-Pérez, Pedro Sáenz-López-Buñuel y Eduardo J. Fernández-Ozcorta 
http://doi.org/10.15359/ree.25-2.17

dichos índices pueden ser considerados como satisfactorios al alcanzar un valor de 041 el RMSEA y .011 el SRMR. Asimismo, el valor de .99 del GFI también es aceptable, ya que es superior a .90. Respecto al X2/g.l., valores entre 2 y 3 representan un ajuste aceptable (Schermelleh-Engel et al., 2003), por lo que los valores obtenidos nos indican que podemos considerar el ajuste satisfactorio al presentar un valor de 2.66. Teniendo en cuenta los resultados obtenidos en relación con los índices de ajuste, se observa que existe un buen ajuste entre el modelo teórico postulado y los datos, por tanto se puede considerar como un modelo adecuado.

La validez convergente fue estudiada a través del análisis de la validez de los indicadores, la cual fue estimada, siguiendo a Bollen (1989), mediante la magnitud de las cargas factoriales. Las saturaciones de los indicadores fueron estadísticamente significativas ( $t \geq|1.96|)$, de manera que todos los indicadores evaluaban el mismo constructo. Asimismo, las cargas factoriales aportaban valores superiores a .60, por lo que se podía aceptar la validez de los indicadores (Lévy Mangin y Varela Mallou, 2006). Por otro lado, los índices de ajuste incrementales fueron adecuados: $\mathrm{NFI}=.996$ y $\mathrm{AGFI}=.986$.

Las correlaciones establecidas entre la relevancia competencial del libro de texto con las subescalas de la escala de motivación educativa (Tabla 4) nos permitieron apreciar la existencia de evidencias externas de la validez de la escala diseñada, ya que se obtuvieron correlaciones positivas y significativas con la regulación externa $(r=.140)$, regulación introyectada ( $r=.300)$, motivación intrínseca al conocimiento $(r=.559)$ y motivación intrínseca al logro $(r=.442)$. Sin embargo, con la amotivación lo hizo de forma negativa y significativamente $(r=-.299)$.

Tabla 4: Estadísticos descriptivos y correlaciones de las variables de estudio

\begin{tabular}{lcccccccccc}
\hline & M & DT & A & C & 1 & 2 & 3 & 4 & 5 & 6 \\
\hline $\begin{array}{l}\text { 1. Relevancia competencial motivacional } \\
\text { de libro de texto ciencias }\end{array}$ & 3.58 & .99 & -.491 & -.355 & - & $-.299^{* *}$ & $.140^{* *}$ & $.300^{* *}$ & $.559^{* *}$ & $.442^{* *}$ \\
2. Amotivación & 1.43 & .64 & 1.624 & 2.161 & - & - & $-.145^{* *}$ & $-.178^{* *}$ & $-.328^{* *}$ & $-.312^{* *}$ \\
3. Regulación externa & 4.55 & .58 & -1.507 & 2.025 & - & - & - & $.396^{* *}$ & $.197^{* *}$ & $.281^{* *}$ \\
4. Regulación introyectada & 4.08 & .74 & -.737 & .159 & - & - & - & - & $.478^{* *}$ & $.549^{* *}$ \\
5. Motivación intrínseca conocimiento & 4.04 & .87 & -.588 & .133 & - & - & - & - & - & $.669^{* *}$ \\
& & & & & & & & & & \\
6. Motivación intrínseca logro & 4.08 & .85 & -.865 & .147 & - & - & - & - & - & - \\
\hline
\end{tabular}

M: media aritmética; DT: Desviación típica; A: Asimetría; C: Curtosis; ${ }^{* *}: \mathrm{p}<.001$.

Nota: Elaboración propia. 
http://doi.org/10.15359/ree.25-2.17

http://www.una.ac.cr/educare

educare@una.ac.cr

\section{Discusión y conclusiones}

El objetivo central de este estudio es proponer un instrumento de evaluación para medir la relevancia del libro de texto de ciencias a nivel motivacional desde una perspectiva competencial. Los resultados obtenidos mediante el procedimiento exploratorio y confirmatorio mostraron una calidad métrica satisfactoria de la escala y un ajuste adecuado del modelo propuesto. En concreto, revelaron la existencia de un único factor formado por cuatro ítems. Estos resultados son congruentes con uno de los postulados principales de la teoría de la autodeterminación. Esta teoría asume que es inherente, a la naturaleza humana, la propensión a sentir curiosidad por el entorno cercano y mostrar interés en aprender y desarrollar el conocimiento que cada persona encuentre significante (Deci y Ryan, 1985; Ryan y Deci, 2019), por lo que puede ser comprensible el hecho de que los cuatro ítems describan un mismo factor. En consecuencia, un contenido con interés para el alumnado (Ames, 1992; Ballester Vila y Sánchez Santamaría, 2011; Cubero Pérez, 2001), la utilidad de lo aprendido en el entorno más próximo (Ballester Vila y Sánchez Santamaría, 2011; Couso Lagarón et al., 2011; de Toledo y Camero, 2015; Ministerio de Educación y Formación Profesional, 2019 ; Pozuelos Estrada, 2007; Rubio Hurtado et al., 2016; Solbes et al., 2007), un aprendizaje que suscite la curiosidad y la necesidad de continuar aprendiendo (Cubero Pérez, 2001; Ministerio de Educación y Formación Profesional, 2019; Ugartetxea, 2002), así como la satisfacción de la curiosidad (Ballester Vila y Sánchez Santamaría, 2011; Cubero Pérez, 2001; Marco Stiefel, 2008; Ugartetxea, 2002), son cuatro aspectos que, unidos, conforman un único constructo capaz de reflejar la relevancia motivacional, desde un enfoque competencial, del libro de texto de ciencias. Asimismo, el análisis sobre la consistencia interna y la estabilidad temporal también mostró unos resultados adecuados, lo que facilita su aplicabilidad en diversos contextos.

Las relaciones entre la escala diseñada y los niveles de motivación proporcionaron las evidencias externas necesarias de la validez del instrumento. En concreto, los resultados revelaron una correlación negativa y significativa con la amotivación, mientras que ocurría justamente lo contrario con el resto de las motivaciones, por tanto, se encontraban en la línea de lo establecido por la teoría de la autodeterminación. Es decir, las personas se encontrarán intrínsecamente motivadas únicamente para tareas con un interés intrínseco por su novedad, desafío o estética (Ryan y Deci, 2000). En este sentido, la investigación de Suárez-Riveiro et al. (2005) ya apuntaba que el valor de la tarea mostraba efectos positivos y significativos sobre la orientación del alumnado hacia el aprendizaje y el desarrollo de capacidades. Asimismo, estudios como el de Alonso Tapia y de la Red Fadrique (2007) ponen de manifiesto que la introducción de tareas más relevantes parece que tiene un efecto positivo sobre la motivación. Mientras que, González y Paoloni (2015) detectaron que la percepción de relevancia de las tareas predijo la implicación y el rendimiento, ya que el alumnado que percibía que se les explicaba mejor la relevancia y utilidad de las tareas, se implicaba más en la asignatura, la consideraban más relevante de cara a su futuro, mostraba más interés por los contenidos y la forma de razonar en ciencias. 
A su vez, las tareas que no presentan novedad, desafío o no tienen una estética interesante no se iniciarán a través de la motivación intrínseca (Deci y Ryan, 1985), necesitan una motivación externa para iniciarlas y mantenerlas (Ryan y Deci, 2019). En este sentido, García-Señorán et al. (2015) han obtenido datos en los que se coincide en que las actividades largas, aburridas o poco interesantes son tomadas por el alumnado de primaria como desmotivadoras a la hora de implicarse en la tarea escolar.

En consecuencia, el valor que el alumnado atribuya a sus actividades influye en su motivación, de manera que, si esta valoración es negativa, difícilmente favorecerá una motivación intrínseca (González y Paoloni, 2015). Mientras que, aparecerá la amotivación cuando la actividad no es valorada (Ryan, 1995). Por ello, una cuestión primordial es conseguir motivar al alumnado para que valore dichas actividades y las realice sin necesidad de presiones externas (Ryan y Deci, 2019). Es decir, una percepción adecuada de la relevancia competencial motivacional del libro de texto favorecía las motivaciones más autodeterminadas, aspecto indispensable para el desarrollo competencial del alumnado (Marco Stiefel, 2008; OCDE, 2006).

Todas las evidencias empíricas analizadas hasta el momento y obtenidas mediante el análisis factorial, de fiabilidad y validez externa completaron la validez del contenido, y permiten deducir que la escala tenía una buena calidad psicométrica. No obstante, como limitación de esta investigación se debe tener en cuenta que en el análisis correlacional se establecen relaciones entre variables sin que esto suponga una relación de causalidad. Sin embargo, estas relaciones contribuyen a perfilar un modelo explicativo, que servirán a su vez como evidencia externa de la validez de la escala diseñada.

La utilidad de proponer instrumentos para evaluar la relevancia motivacional de los aspectos contextuales implicados en la motivación se basa en el acercamiento que nos ofrece a la comprensión de esta, la cual es indispensable para el desarrollo competencial del alumnado. En concreto en este caso, permitirá valorar, desde la perspectiva del estudiantado, la relevancia de uno de los recursos más fuertemente vinculados a las clases de ciencias en su desarrollo competencial, y centrarse, en este caso, en su vertiente motivacional. Por tanto, ayudará al profesorado a tomar las decisiones metodológicas apropiadas relacionadas con este recurso.

Una de las sugerencias didácticas, extraída como consecuencia de esta investigación, pasaría por utilizar el libro teniendo en cuenta los aspectos que se han referenciado en la escala como elementos claves relacionados con el desarrollo competencial desde un enfoque motivacional. Es decir, utilizar el libro de manera que se fomente la curiosidad del alumnado, colocando el foco de atención sobre contenidos interesantes y transmitiendo una visión de utilidad de lo trabajado mediante la resolución de las inquietudes del alumnado y su uso en la vida cotidiana.

En cuanto a futuras líneas de investigación, resultaría interesante abordar un estudio sobre la relación entre la relevancia competencial motivacional del libro de texto de ciencias con otras variables motivacionales como el clima de aula, las necesidades psicológicas básicas y el rendimiento académico, así como indagar sobre las diferencias entre géneros o etapas educativas. 
http://doi.org/10.15359/ree.25-2.17

http://www.una.ac.cr/educare

educare@una.ac.cr

\section{Declaración de Material complementario}

Este artículo tiene disponible, como material complementario:

-La versión preprint del artículo en https://doi.org/10.5281/zenodo.4066897

\section{Referencias}

Abad, F. J., Olea, J., Ponsoda, V. y García, C. (2011). Medición en ciencias sociales y de la salud. Proyecto editorial: Metodología de las ciencias del comportamiento y de la salud. Síntesis.

Alonso Tapia, J. y de la Red Fadrique, I. (2007). Evaluar "para" el aprendizaje, aprender para estar motivado: El orden de los factores sí afecta al producto. Revista Española de Orientación y Psicopedagogía, 18(2), 241-253. http://revistas.uned.es/index.php/reop/article/ view/11315

Ames, C. (1992). Achievement goals, motivational climate and motivational processes. En G. C. Roberts (Ed.), Motivational in sport and exercise (161-176). Human Kinetics Books.

Ballester Vila, M. G. y Sánchez Santamaría, J. (2011). La dimensión pedagógica del enfoque de competencias en educación obligatoria. Ensayos. Revista de la Facultad de Educación de Albacete, 26, 17-34. https://revista.uclm.es/index.php/ensayos/article/view/69/32

Bollen, K. A. (1989). Structural equations with latent variables. John Wiley \& Sons. https://doi. org/10.1002/9781118619179

Busquets, T., Silva, M. y Larrosa, P. (2016). Reflexiones sobre el aprendizaje de las ciencias naturales: Nuevas aproximaciones y desafíos. Estudios Pedagógicos, 42(especial), 117-135. https://dx.doi.org/10.4067/S0718-07052016000300010

Castro-Pérez, M. y Morales-Ramírez, M. E. (2015). Los ambientes de aula que promueven el aprendizaje, desde la perspectiva de los niños y niñas escolares. Revista Electrónica Educare, 19(3), 1-32. https://doi.org/10.15359/ree.19-3.11

Coronas Cabrero, M. (1995). La prensa y las ciencias naturales. Comunicar, 2(4), 75-78. https://doi. org/10.3916/C04-1995-14

Couso Lagarón, D., Jiménez Aleixandre, M. P., López-Ruiz, J., Mans Teixidó, C., Rodríguez Espinosa, J. M., Sanmartí Puig, N. (2011). Informe Enciende. Enseñanza de las ciencias en la didáctica escolar para edades tempranas en España. COSCE. https://www.cosce.org/pdf/Informe ENCIENDE.pdf 
http://doi.org/10.15359/ree.25-2.17

Cubero Pérez, R. (2001). Motivación e interés por los contenidos escolares. Aula de Innovación educativa, 101, 28-31. https://www.grao.com/es/producto/motivacion-e-interes-por-loscontenidos-escolares

Deci, E. L. y Ryan, R. M. (1985). Intrinsic motivation and self-determination in human behavior. Plenum. https://doi.org/10.1007/978-1-4899-2271-7

de Toledo, M. O. y Camero, R. E. (2015). Desarrollo de cinco recursos con enfoque CTS para la enseñanza desistema respiratorio, circulatorioy digestivo. Revista de Investigación, 39(85),6391. http://ve.scielo.org/scielo.php?script=sci arttext\&pid=S1010-29142015000200005\&ln $\mathrm{g}=\mathrm{es} \& \operatorname{tn} \mathrm{ng}=\mathrm{es}$.

García-Señorán, M. del M., González González, S. G.y Soto Carballo, J.G (2015). Estudio exploratorio de intereses y motivación para la ejecución de tareas en alumnado de educación primaria de la provincia de Pontevedra. Revista de Investigación en Educación, 13(2), 256-270. http:// reined.webs.uvigo.es/index.php/reined/article/view/233

González, A. y Paoloni, P.-V. (2015). Implicación y rendimiento en física: El papel de las estrategias docentes en el aula, y el interés personal y situacional del alumnado. Revista de Psicodidáctica, 20(1), 25-45. https://doi.org/10.1387/RevPsicodidact.11370

Howard, J. L., Bureau, J., Guay, F., Chong, J. X. Y., y Ryan, R. M. (2021). Student motivation and associated outcomes: A meta-analysis from self-determination theory. Perspectives on Psychological Science. https://doi.org/10.1177/1745691620966789

Lévy Mangin, J.-P. (Dir.) y Varela Mallou, J. (Coord.) (2006). Modelización con estructuras de covarianzas en ciencias sociales. Netbiblo.

Marco Stiefel, B. (2008). Competencias básicas. Hacia un nuevo paradigma educativo. Narcea.

Ministerio de Educación y Formación Profesional. (2019). PISA 2018. Programa para la evaluación internacional de los estudiantes. Informe español. Secretaría General Técnica. https://sede. educacion.gob.es/publiventa/descarga.action?f codigo agc $=20372$

Nuñez, J. L., Martín-Albo, J., Navarro, J. G. y Suárez, Z. (2010). Adaptación y validación de la versión española de la Escala de motivación educativa en estudiantes de educación secundaria postobligatoria. Estudios de Psicología, 31(1), 89-100. https://doi. org/10.1174/021093910790744590

Occelli, M. y Valeiras, N. (2013). Los libros de texto de ciencias como objeto de investigación: Una revisión bibliográfica. Enseñanza de las Ciencias, 31(2), 133-152. https://doi.org/10.5565/ rev/ec/v31n2.761 
http://doi.org/10.15359/ree.25-2.17

http://www.una.ac.cr/educare

educare@una.ac.cr

Organización para la Cooperación y el Desarrollo Económicos. (2006). PISA 2006. Marco de la evaluación. Conocimientos y habilidades en ciencias, matemáticas y lectura. Autor. https:// www.oecd.org/pisa/publicacionesdepisaenespaol.htm

Perrenoud, P. (2012). Cuando la escuela pretende preparar para la vida. ¿Desarrollar competencias o enseñar otros saberes? Graó.

Pintrich, P. R. (1999). The role of motivation in promoting and sustaining self-regulated learning. International Journal of Educational Research 31(6), 459-470. https://www.sciencedirect. com/science/article/pii/S0883035599000154

Pozuelos Estrada, F. J. (2007). Trabajo por proyectos en el aula: Descripción, investigación y experiencias. M.C.E.P

Rubio Hurtado, M. J., Ruiz Bueno, A. y Martínez-Olmo, F. (2016). Percepción del alumnado sobre la utilidad de las actividades de aprendizaje para desarrollar competencias. Revista de Investigación Educativa, 34(1), 221-240. https://doi.org/10.6018/rie.34.1.225131

Ryan, R. M. (1995). Psychological needs and the facilitation of integrative processes. Journal of Personality, 63(3), 397-427. https://doi.org/10.1111/j.1467-6494.1995.tb00501.x

Ryan, R. M. y Deci, E. L. (2000). Self-determination theory and the facilitation on intrinsic motivation, social development, and well-being. American Psychologist, 55(1), 68-78. https://doi.org/10.1037/0003-066X.55.1.68

Ryan, R. M.y Deci, E. L. (2017). Self-determination theory: Basic psychological needs in motivation, development, and wellness. Guilford.

Ryan, R. M., y Deci, E. L. (2019). Brick by brick: The origins, development, and future of selfdetermination theory. En A. J. Elliot (Ed.), Advances in motivation science (Vol. 6, pp. 111156). Elsevier. https://doi.org/10.1016/bs.adms.2019.01.001

Ryan, R. M. y Deci, E. L. (2020). Intrinsic and extrinsic motivation from a self-determination theory perspective: Definitions, theory, practices, and future directions. Contemporary Educational Psychology, 61, 1-31.https://doi.org/10.1016/j.cedpsych.2020.101860

Simpson, E. H. y Balsam, P. D. (2016). The behavioral neuroscience of motivation: An overview of concepts, measures, and translational applications. Current Topics in Behavioral Neurosciences, 27, 1-12. https://doi.org/10.1007/7854 2015402

Schermelleh-Engel, K., Moosbrugger, H. y Müller, H. (2003). Evaluating the fit of structural equation models: Tests of significance and descriptive goodness-of-fit measures. Methods of Psychological Research, 8(2), 23-74. https://www.researchgate.net/ publication/251060246 Evaluating the Fit of Structural Equation Models Tests of Significance_and_Descriptive_Goodness-of-Fit_Measures 
http://doi.org/10.15359/ree.25-2.17

http://www.una.ac.cr/educare educare@una.ac.cr

Solbes, J., Montserrat, R. y Furió, C. (2007). El desinterés del alumnado hacia el aprendizaje de la ciencia: Implicaciones en su enseñanza. Didáctica de las Ciencias Experimentales y Sociales, 21, 91-117. https://ojs.uv.es/index.php/dces/article/view/2428/1973

Suárez Riveiro, J. M., Fernández Suárez, A. P. y Anaya Nieto, D. (2005). Un modelo sobre la determinación motivacional del aprendizaje autorregulado. Revista de Educación, 338, 295-306. $\quad$ http://www.educacionyfp.gob.es/revista-de-educacion/numeros-revistaeducacion/numeros-anteriores/2005/re338/re338-17.html

Ugartetxea, J. (2002). La metacognición, el desarrollo de la autoeficacia y la motivación escolar. Revista de Psicodidáctica, 13, 49-73. https://www.ehu.eus/ojs/index.php/psicodidactica/ article/view/135

Vallerand, R. J. (1997). Toward a hierarchical model of intrinsic and extrinsic motivation. Advances in Experimental Social Psychology, 29, 271-360. https://doi.org/10.1016/S00652601(08)60019-2

Velicer, W. F. y Fava, J. L. (1998). Effects of variable and subject sampling on factor pattern recovery. Psychological Methods, 3(2), 231-251. https://doi.org/10.1037/1082-989X.3.2.231 